\title{
A rare cause of hepatomegaly and dyslipidemia: lysosomal acid lipase deficiency
}

\author{
Berrak Bilginer Gürbüz ${ }^{1 \oplus}$, İlker Güney ${ }^{2 \oplus}$, Fatma Derya Bulut ${ }^{1 \oplus}$, Okan Dilek ${ }^{3 \oplus}$ \\ Divisions of ${ }^{1}$ Pediatric Metabolism, ${ }^{2}$ Medical Genetics and ${ }^{3}$ Radiology, Adana City Education and Training Hospital, Adana, Turkey.
}

\begin{abstract}
Background. Lysosomal acid lipase deficiency (LAL-D), also known as cholesteryl ester storage disease or Wolman disease, is a multi-systemic autosomal recessive genetic disorder caused by mutations in the lysosomal acid lipase gene (LIPA).

Case. A 14-year-old female patient was diagnosed as LAL-D with the findings of hepatomegaly, splenomegaly, elevated liver enzyme levels, and abnormal lipid profile. Her sister had similar laboratory and ultrasonographic findings. Both siblings had a homozygous c.894 G>A mutation in the LIPA gene, and their parents were heterozygous for this mutation.

Conclusions. This case is one of the similar reports in the literature regarding clinical, biochemical, and genetic findings. It is well-known that LAL-D has overlapping clinical manifestations, and early diagnosis is quite challenging. Therefore, most patients die in the first year of life. After the determination of novel mutations in LAL-D patients, it is thought that LAL-D can present with heterogeneous signs and symptoms.
\end{abstract}

Key words: Lysosomal acid lipase (LAL), Lysosomal acid lipase deficiency (LALD), cholesterol ester storage disease (CESD).

Lysosomal acid lipase deficiency (LAL-D), also named as cholesteryl ester storage disease and Wolman disease, is a multi-systemic autosomal recessive genetic disorder caused by mutations in the lysosomal acid lipase gene (LIPA) that encodes the enzyme lysosomal acid lipase (LAL). ${ }^{1,2}$ LAL-D is responsible for the accumulation of triglycerides (TG) and progressive cholesteryl esters (CE) in most cells, including liver and spleen. ${ }^{3}$ LAL-D is historically divided into two chief clinical phenotypes: earlyonset Wolman disease (WD) and late-onset cholesteryl ester storage disease (CESD). ${ }^{4} \mathrm{WD}$ is a rare and rapidly progressive form that unveils in the first week of life. It leads to severe earlyonset disorders, including chronic malnutrition, hepatosplenomegaly, adrenal calcification, steatorrhea, emesis, and multiorgan failure. ${ }^{5}$

$凶$ Berrak Bilginer Gürbüz

berrakgurbuz@yahoo.com.tr

Received 13th April 2020, revised 14th May 2020, accepted 16th May 2020.
Most of the patients with WD die within the first year of life. On the other hand, CESD is an unrecognized form that manifests later (between 3-15 years of age). It also leads to a broad spectrum of clinical manifestations, including accelerated atherosclerosis, hepatic steatosis, hepatosplenomegaly, and dyslipidemia. The incidence of LAL-D is estimated to be between $1 / 40.000$ and $1 / 300.000 .^{6}$

Additionally, a $36 \mathrm{~kb}$ LIPA gene is located on chromosome 10q22.2 and contains 10 exons. According to the literature, more than 60 LIPA defects have been identified in LAL-D patients, and new studies are still describing other mutations. The most common LIPA defect is c. $894 \mathrm{G}>\mathrm{A} .{ }^{3}$ On the other hand, progressive TG and CE accumulation is associated with characteristic liver damage, increased liver transaminases, serum low-density lipoprotein cholesterol (LDL-c) and triglycerides, and normal/low high-density lipoprotein cholesterol (HDL-c) levels. ${ }^{2}$ Many patients with 
LAL-D suffer from accelerated atherosclerosis, hepatosplenomegaly, cirrhosis, liver fibrosis, and liver failure. ${ }^{7}$ Enzyme activity assays from dried blood, and confirm by genetic sequencing in whole blood are the most preferred diagnostic techniques.

In this study, we report siblings with clinical and biochemical aspects of LAL-D with homozygous c.894G>A mutation in the LIPA gene.

\section{Case Reports}

A 14-year-old female patient was admitted to our hospital with abdominal distension due to hepatomegaly. The proband had a birth history of normal weight and height. Her family history revealed that the parents were first-degree cousins. On physical examination, the patient had a weight of $55.2 \mathrm{~kg}$ [ 0.27 standard deviation (SD)], a height of $148.4 \mathrm{~cm}$ (-2.02 SD), the liver was about $1 \mathrm{~cm}$ palpable, and other systemic examinations were normal.

Theinitiallaboratory tests showed increased liver enzymes, including alanine aminotransferase (ALT) $(63.1 \mathrm{IU} / \mathrm{L}$; reference value < $50 \mathrm{IU} / \mathrm{L})$, aspartate aminotransferase (AST) $(53.8 \mathrm{IU} / \mathrm{L}$; reference value $<50 \mathrm{IU} / \mathrm{L})$, gamma-glutamyl transferase (GGT) (48 IU/L; reference value < $40 \mathrm{IU} / \mathrm{L}$ ), and lactate dehydrogenase (LDH) 195 U/L (reference value $<247$ IU/L). Furthermore, her creatine kinase (CK) level was $85 \mathrm{U} / \mathrm{L}$ (reference value $<145 \mathrm{U} / \mathrm{L}$ ) total bilirubin value was $1.48 \mathrm{mg} / \mathrm{dl}$ (reference value $<1.2 \mathrm{mg}$ / dl) and direct bilirubin value was $0.15 \mathrm{mg} / \mathrm{dl}$ (reference value $<0.2 \mathrm{mg} / \mathrm{dl}$ ). Dyslipidemia was detected in addition to elevated liver enzymes. Her serum lipid profile findings showed a total cholesterol (TC) level of $280 \mathrm{mg} / \mathrm{dl}$, a lowdensity lipoprotein (LDL) level of $210.5 \mathrm{mg} /$ $\mathrm{dl}$, and a high-density lipoprotein (HDL) level of $39.5 \mathrm{mg} / \mathrm{dl}$, triglyceride level of $295 \mathrm{mg} / \mathrm{dl}$ which were compatible with dyslipidemia.

The abdominal ultrasound demonstrated grade 1 hepatosteatosis with hepatomegaly and splenomegaly(Fig.1). Therewerenopathological findings on the echocardiography evaluation of the patient. At this stage of the investigation, LAL-D was suspected. The LAL activity was measured from a dried blood spot card, and LAL-D was diagnosed by verifying low acid lipase activity $(<0.02 \mathrm{nmol} / \mathrm{punch} / \mathrm{hr}$; reference range: $0.37-2.30$ ). Confirmatory diagnosis of LAL-D was performed with genetic sequencing, which showed a pathological homozygous mutation in c.894G >A. After the diagnosis of LAL-D was confirmed, her three year old sister who had the same complaints was evaluated. It was detected that the younger sister had similar laboratory and ultrasonographic findings. Her height was $91.8 \mathrm{~cm}$ (-0.9 SD), and her weight was $15.2 \mathrm{~kg}$ (0.56 SD). Physical examination was normal except for about $2 \mathrm{~cm}$ palpable liver. She also had increased liver enzymes (ALT: $56.1 \mathrm{IU} / \mathrm{L}, \mathrm{AST}$ : $79.7 \mathrm{IU} / \mathrm{L})$, and dyslipidemia (TC: $279 \mathrm{mg} / \mathrm{dl}$, LDL: $218 \mathrm{mg} / \mathrm{dl}$, HDL: $41 \mathrm{mg} /$ $\mathrm{dl}$, and triglyceride: $111 \mathrm{mg} / \mathrm{dl}$ ). Furthermore, her abdominal ultrasound was compatible with hepatomegaly and splenomegaly (Fig. 1). She had a normal echocardiography evaluation.

Both siblings had a homozygous c.894G $>$ A mutation in the LIPA gene, and their parents
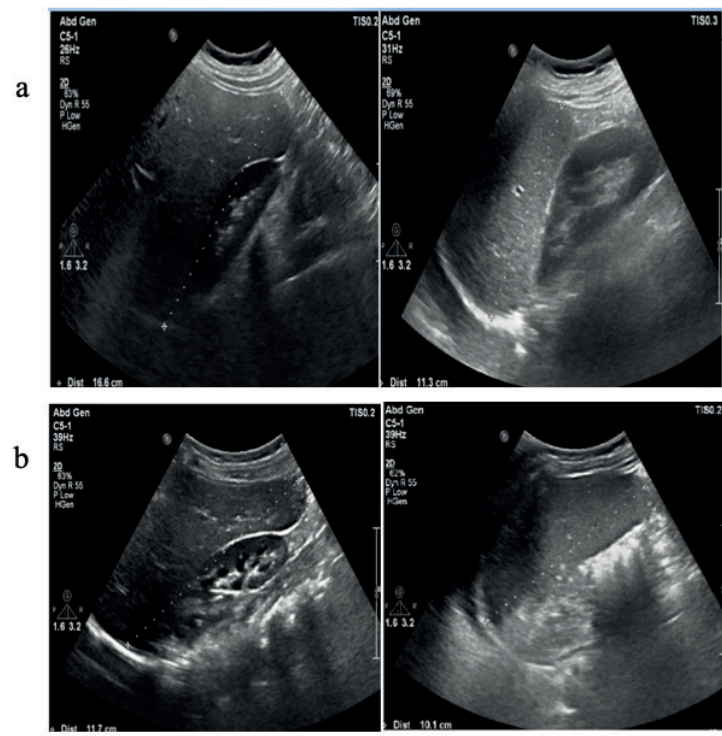

Fig. 1. Ultrasound images showing the maximum lengths of patients' (a:index case, b:sibling) liver and spleen. 
were heterozygous for this mutation (Fig. 2A, 2B). However, there were no phenotypical changes in both siblings.

A low-fat diet and omega-3 supportive therapy were started both patients. We planned to start Sepelipase alpha enzyme treatment, which has proven efficacy in LAL-D patients. However, the drug could not be obtained due to the health practices policy in our country. In addition, statin therapy was added to the proband patient due to dyslipidemia. Despite the treatments applied in the follow-up of the patients, no positive response was observed, especially in terms of dyslipidemia, and liver enzyme elevations also persisted.

The parents of the patient were informed, and written and oral consent was obtained according to the principles of the Helsinki Declaration.

\section{Discussion}

LAL-D is known as a rare lipid storage disease with early mortality and significant morbidity. Its incidence is higher among individuals with Iranian-Jewish ancestry $(1: 4200){ }^{8}$ The natural course of the late-onset form of the disease results in inflammation, and the findings from the hepatomegaly and splenomegaly biopsy specimens are indicative of CESD. ${ }^{9}$ There is a wide range of signs and symptoms of CESD, including vomiting, diarrhea, low level of HDL cholesterol, liver steatosis, growth failure, hypercholesterolemia, as well as elevated AST, ALT, and GGT levels. ${ }^{10}$ Additionally, according to the Human Gene Mutation Database, the most common LIPA mutation in CESD patients is c. $894 \mathrm{G}>\mathrm{A}$, which is a LIPA gene exon 8 splice junction mutation. ${ }^{6}$

In this study, we presented a patient with lateonset LAL-D, who had clinical, enzymatic, and lipid profile abnormalities. Increased AST, ALT, GGT, TC, LDL, together with decreased HDL and acid lipase activity, were detected in our patient. An exon 8 c.894G $>$ A LIPA gene mutation was confirmed. Due to its low incidence in society and having similar signs and symptoms with other diseases, LAL-D is hard to diagnose. ${ }^{7}$ Some studies suggested that LAL enzymatic activities should be measured in patients with abnormal lipid and enzyme profiles such as elevated LDL, TG, TC, AST, ALT, and reduced HDL. ${ }^{6,711}$ Similar reports and suggestions are available in the literature. Kuranobu et al. ${ }^{9}$ reported a case of an 11-yearold male CESD patient with abnormal lipid and

A

B

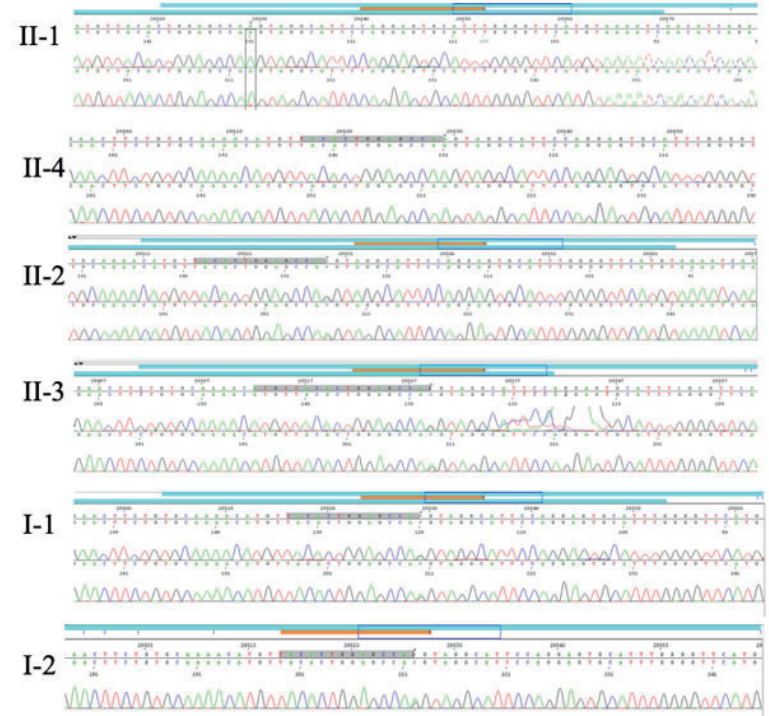

Fig. 2A, 2B. Pedigree and sequence analyses of family. Both siblings (II-1, II-4) homozygous, parents and other two siblings (I-1, I-1, II-2, II-3) were heterozygous for this mutation. 
enzyme profiles and a novel mutation in the LIPA gene.

Similarly, Benevides et al. ${ }^{12}$ reported a case series with seven patients with LAL-D (both WD and CESD). They detected abnormal lipid and enzyme profiles in all patients, as well as other expected symptoms. Additionally, diagnostic images such as liver/spleen ultrasound (US) and biopsy are necessary to show the changes in organ morphology. As seen in our case, hepatomegaly and splenomegaly were detected by ultrasonography. Hepatic morphological changes such as microvesicular steatosis with Kupffer cell involvement, fibrosis, and cirrhosis were reported by other researchers. ${ }^{10,12-14}$

On the other hand, homozygous LIPA gene mutation of c. $894 \mathrm{G}>\mathrm{A}$ has been detected in more than $50 \%$ of patients with late-onset LAL-D. The same gene mutation was also detected in our patient. This mutation occurs in exon 8 of the LIPA gene that encodes a mutant enzyme with no residual LAL activity. ${ }^{15}$ However, some researchers reported novel mutations in LAL-D patients such as c.607 G>C, c.791 T>C, c.266 T>C, and c.67 G>A.9.12

In conclusion, this rare disease should be suspected in presentations with hepatomegaly, fatty liver, and dyslipidemia. Our case is one of the similar reports in the literature regarding clinical, biochemical, and genetic findings. It is well-known that LAL-D has overlapping clinical manifestations, and early diagnosis is quite challenging. Therefore, most patients die in the first year of the disease. After the determination of novel mutations in LAL-D patients, it is thought that LAL-D can present with heterogeneous signs and symptoms, which might facilitate making an accurate diagnosis.

\section{REFERENCES}

1. Cunha-Silva M, Mazo DFC, Correa BR, et al. Lysosomal acid lipase deficiency leading to liver cirrhosis: a case report of a rare variant mutation. Ann Hepatol 2019; 18: 230-235.
2. Ikari N, Shimizu A, Asano T. Lysosomal acid lipase deficiency in Japan: a case report of siblings and a literature review of cases in Japan. J Nippon Med Sch 2018; 85: 131-137.

3. Bychkov IO, Kamenets EA, Filatova AY, et al. The novel synonymous variant in LIPA gene affects splicing and causes lysosomal acid lipase deficiency. Mol Genet Metab 2019; 127: 212-215.

4. Soll D, Spira D, Hollstein T, et al. Clinical outcome of a patient with lysosomal acid lipase deficiency and first results after initiation of treatment with Sebelipase alfa: a case report. Mol Genet Metab Rep 2019; 20: 100479.

5. Balwani M, Breen C, Enns GM, et al. Clinical effect and safety profile of recombinant human lysosomal acid lipase in patients with cholesteryl ester storage disease. Hepatology 2013; 58: 950-957.

6. Botero V, Garcia VH, Gomez-Duarte C, et al. Lysosomal acid lipase deficiency, a rare pathology: the first pediatric patient reported in Colombia. Am J Case Rep 2018; 19: 669-672.

7. Reiner Z, Guardamagna O, Nair D, et al. Lysosomal acid lipase deficiency--an under-recognized cause of dyslipidaemia and liver dysfunction. Atherosclerosis 2014; 235: 21-30.

8. Wierzbicka-Rucinska A, Janczyk W, Lugowska A, Lebensztejn D, Socha P. Diagnostic and therapeutic management of children with lysosomal acid lipase deficiency (LAL-D). Review of the literature and own experience. Dev Period Med 2016; 20: 212-215.

9. Kuranobu N, Murakami J, Okamoto K, et al. Cholesterol ester storage disease with a novel LIPA mutation (L264P) that presented massive hepatomegaly: a case report. Hepatol Res 2016; 46: 477-482.

10. Valayannopoulos V, Mengel E, Brassier A, Grabowski G. Lysosomal acid lipase deficiency: expanding differential diagnosis. Mol Genet Metab 2017; 120: 62-66.

11. Sreekantam S, Nicklaus-Wollenteit I, Orr J, et al. Successful long-term outcome of liver transplantation in late-onset lysosomal acid lipase deficiency. Pediatr Transplant 2016; 20: 851-854.

12. Benevides GN, Miura IK, Person NC, et al. Lysosomal acid lipase deficiency in Brazilian children: a case series. J Pediatr (Rio J) 2019; 95: 552-558.

13. Curiati MA, Kyosen SO, Pereira VG, Patricio FRDS, Martins AM. Lysosomal acid lipase deficiency: report of five cases across the age spectrum. Case Rep Pediatr 2018; 2018: 4375434. 
14. Tommaso AMAD, Barra FFDC, Hessel G, Moreno CA, Giugliani R, Escanhoela CAF. Importance of liver biopsy in the diagnosis of lysosomal acid lipase deficiency: a case report. Rev Paul Pediatr 2018; 36: 113-116.
15. Scott SA, Liu B, Nazarenko I, et al. Frequency of the cholesteryl ester storage disease common LIPA E8SJM mutation (c.894G>A) in various racial and ethnic groups. Hepatology 2013; 58: 958-965. 